\title{
Suficiência amostral para estudos de impacto ambiental sobre a comunidade de macroinvertebrados bentônicos em arrozais irrigados
}

\author{
Sample sufficiency for environmental impact studies on benthic macroinvertebrate \\ community in irrigated rice fields
}

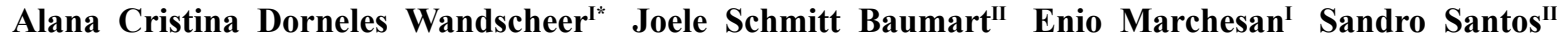
Luis Antonio de Avila ${ }^{\mathrm{III}}$ Camille Flores Soares ${ }^{\mathrm{I}}$ Mateus Marques Pires $^{\mathrm{IV}}$
\end{abstract}

\begin{abstract}
O objetivo deste estudo foi determinar a suficiência amostral para estudos de impacto ambiental sobre a comunidade de macroinvertebrados bentônicos em arrozais irrigados (Oryza sativa L.). Amostragens de solo foram realizadas com auxilio de coletor cilíndrico, nos anos agrícolas 2007/08 e 2012/13. As amostras foram triadas e os espécimes identificados até o nível de família. Os dados foram submetidos à análise da curva do coletor e os indices de diversidade de Shannon e equabilidade de Pielou foram calculados para o total de amostras e o número de amostras definido pela curva do coletor, em cada ano amostral. Considerando o método de amostragem verificado no presente trabalho, indica-se um mínimo de 12 réplicas para se obter o maior número de famílias sem perda de informação, quando em estudos de impacto ambiental sobre a comunidade de macroinvertebrados bentônicos em área de cultivo de arroz irrigado.
\end{abstract}

Palavras-chave: curva do coletor, amostragem, indices de diversidade, invertebrados aquáticos.

\section{ABSTRACT}

The objective of this study was to determine the sample sufficiency in environmental impact studies on the benthic macroinvertebrate community in irrigated rice fields (Oryza sativa L.). Soil samplings were conducted with a cylindrical collector in the agricultural years of 2007/08 and 2012/13. Individuals were sorted out and identified up to family level. Data were analyzed through the collector curve, Shannon's Diversity and Pielou's Evenness Index were calculated for the total number of samples and the number of samples defined by the collector curve in each year. Considering the results observed in this study, we indicate a minimum of 12 replicates to obtain the largest number of families without loss of information, in environmental impact studies on benthic macroinvertebrate community in irrigated rice fields.

Key words: collector curve, sampling, diversity indices, aquatic invertebrates.

Macroinvertebrados bentônicos são organismos que habitam os substratos de fundo nos habitats de água doce, ao menos parte do seu ciclo de vida, e que podem ser retidos em peneira de malha $\geq 200$ a $500 \mu \mathrm{m}$ (ROSENBERG \& RESH, 1993), representados por espécies de Insecta, Annelida, Nemertinea, Crustacea, Mollusca e alguns Turbellaria e Bryozoa (KUHLMANN et al., 2012). Tais organismos ocupam importante posição na fauna aquática dos arrozais irrigados (BARBOUR et al., 1999), auxiliando na fertilidade do solo da lavoura, na ciclagem de nutrientes, além do controle biológico de insetos pragas da cultura (ROGER et al., 1991).

Estudos de impacto ambiental em comunidades aquáticas requerem métodos apropriados para se obter informações precisas sobre mudanças na diversidade e estrutura das comunidades biológicas. A amostragem é necessária porque não é possível acessar a totalidade de um dado universo amostral, sendo necessário avaliar se o tamanho da amostra é suficiente para uma dada precisão requerida

IPrograma de Pós-graduação em Agronomia, Departamento de Fitotecnia, Universidade Federal de Santa Maria (UFSM), Avenida Roraima, 1000, Bairro Camobi, 97105-900, Santa Maria, RS, Brasil. E-mail: alanacdw@hotmail.com. *Autor para correspondência "Programa de Pós-graduação em Biodiversidade Animal, UFSM, Santa Maria, RS, Brasil. IIIPrograma de Pós-graduação em Fitossanidade, Universidade Federal de Pelotas (UFPel), Pelotas, RS, Brasil.

IV Programa de Pós-graduação em Biologia, Escola Politécnica, Universidade do Vale do Rio dos Sinos (UNISINOS), São Leopoldo, RS, Brasil. 
e, portanto, a avaliação de suficiência amostral é uma ferramenta importante para este estudo (PILLAR, 2004). Segundo CAIN (1938), a suficiência amostral é atingida quando um incremento de $10 \%$ no tamanho da amostra corresponde a um incremento menor ou igual a 10\% no número de espécies levantadas.

Segundo SILVA (2012), poucos trabalhos têm sido feitos para determinar a suficiência amostral em estudos com invertebrados do solo. Considerando a necessidade de pesquisas sobre o esforço amostral requerido para estudos de impacto ambiental, o objetivo do presente trabalho é definir a suficiência amostral requerida para ensaios com macroinvertebrados bentônicos em área de cultivo de arroz irrigado.

$\mathrm{O}$ estudo foi realizado em área experimental de várzea, Universidade Federal de Santa Maria, nos anos agrícolas de 2007/08 e 2012/13. Ensaios a campo foram instalados a fim de simular o ambiente da lavoura de arroz irrigado. As parcelas experimentais apresentaram área de $48 \mathrm{~m}^{2}$ $(2007 / 08)$ e $10 \mathrm{~m}^{2}(2012 / 13)$, onde foram semeadas as cultivares 'IRGA 422 CL' (2007/08) e 'PUITA INTA CL' (2012/13), respectivamente. Amostras de solo foram coletadas após o terceiro dia de entrada de água nas parcelas, as quais permaneceram com lâmina de água de $10 \mathrm{~cm}$.

As coletas de solo seguiram dois transectos que se cruzavam no meio das parcelas, sendo que, em cada transecto, foram coletadas 15 amostras a uma distância de aproximadamente $75 \mathrm{~cm}(2007 / 08)$ e 10 amostras a uma distância de aproximadamente $48 \mathrm{~cm}$ (2012/13). As amostragens foram realizadas com auxílio de um cilindro coletor com área de $0,01 \mathrm{~m}^{2}$ a uma profundidade de $10 \mathrm{~cm}$. Após as coletas, as amostras foram lavadas em tamises de 0,25 (2007/08) e $0,50 \mathrm{~mm}(2012 / 13)$ e acondicionadas em frascos plásticos, coradas com "rosa de Bengala" e fixadas com álcool etílico absoluto. No laboratório, o material foi triado e identificado até o nível taxonômico de família (FERNÁNDEZ \& DOMÍNGUEZ, 2001).

A suficiência amostral foi avaliada pela construção da curva do coletor, utilizando-se 100 randomizações. Cada ponto foi considerado uma unidade amostral ( $\mathrm{n} 1=30$ amostras; $\mathrm{n} 2=20$ amostras). A análise foi realizada no programa estatístico EstimateS 8.2 (COLWELL, 2009). A diversidade e a abundância proporcional dos organismos foram avaliadas para o número total de amostras coletadas e o número de amostras obtido na análise de suficiência amostral, através dos índices de Shannon (H') e equabilidade de Pielou (J').

Um total de 3443 indivíduos foi coletado nos dois anos amostrais, distribuídos nos filos
Arthropoda, Mollusca, Annelida e Nematoda. Na safra 2007/08, o filo mais abundante foi Arthropoda, que compôs cerca de $60 \%$ da densidade total encontrada. Na safra 2012/13, aproximadamente 89\% dos organismos corresponderam ao Filo Anellida.

Segundo SILVEIRA (2010), a melhor forma de se avaliar o esforço de amostragem é através da construção da curva do coletor. Este método tem sido muito utilizado no Brasil para indicar a suficiência amostral, um conceito quantitativo para informar se a amostra utilizada é representativa da comunidade em estudo. Quanto maior o tamanho da amostra, maior o número de espécies que poderá ser encontrado, mas a uma taxa decrescente, até o ponto em que a curva estabiliza e torna-se horizontal (SCHILLING \& BATISTA, 2008). Uma vez que a assíntota da curva é atingida, nenhuma amostragem adicional produzirá adição de espécies (GOTELLI \& COLWELL, 2010).

Com os resultados obtidos, identificouse que a suficiência amostral para coleta de macroinvertebrados bentônicos foi atingida após a $11^{\mathrm{a}}$ amostra no primeiro ano de estudo (2007/08) e $10^{\mathrm{a}}$ amostra no segundo ano de estudo (2012/13), quando já haviam aparecido os 15 e 12 táxons identificados nas amostras, respectivamente (Figura 1).

A diversidade é um dos conceitos fundamentais no estudo de comunidades e diversos métodos para sua mensuração estão disponíveis, dentre eles o uso dos índices de diversidade que combinam dois atributos de uma comunidade biológica: o número de espécies e sua equabilidade (MELO et al., 2008).

O índice de diversidade de Shannon (H') e o índice de equabilidade de Pielou (J') apresentaram valores próximos para o número de amostras totais e a suficiência amostral, para cada ano estudado, indicando que a análise da suficiência amostral foi eficaz para uma representação significativa da comunidade de macroinvertebrados bentônicos. Para o ano de 2007/08, o índice de Shannon para amostras totais foi de 1,188 e 1,156 para a suficiência amostral. $\mathrm{O}$ índice de equabilidade de Pielou foi 0,438 para o número de amostras totais e 0,427 para a suficiência amostral. Para o ano de 2012/13, o índice de Shannon foi de 0,496 para o número de amostras totais e 0,413 para a suficiência amostral e o índice de equabilidade de Pielou apresentou o valor de 0,199 para o número de amostras totais e 0,166 para a suficiência amostral.

As curvas de acumulação de espécies, bem como os estimadores de riqueza, fornecem subsídios para a tomada de decisões em relação à riqueza taxonômica e a conservação da biodiversidade, sendo 


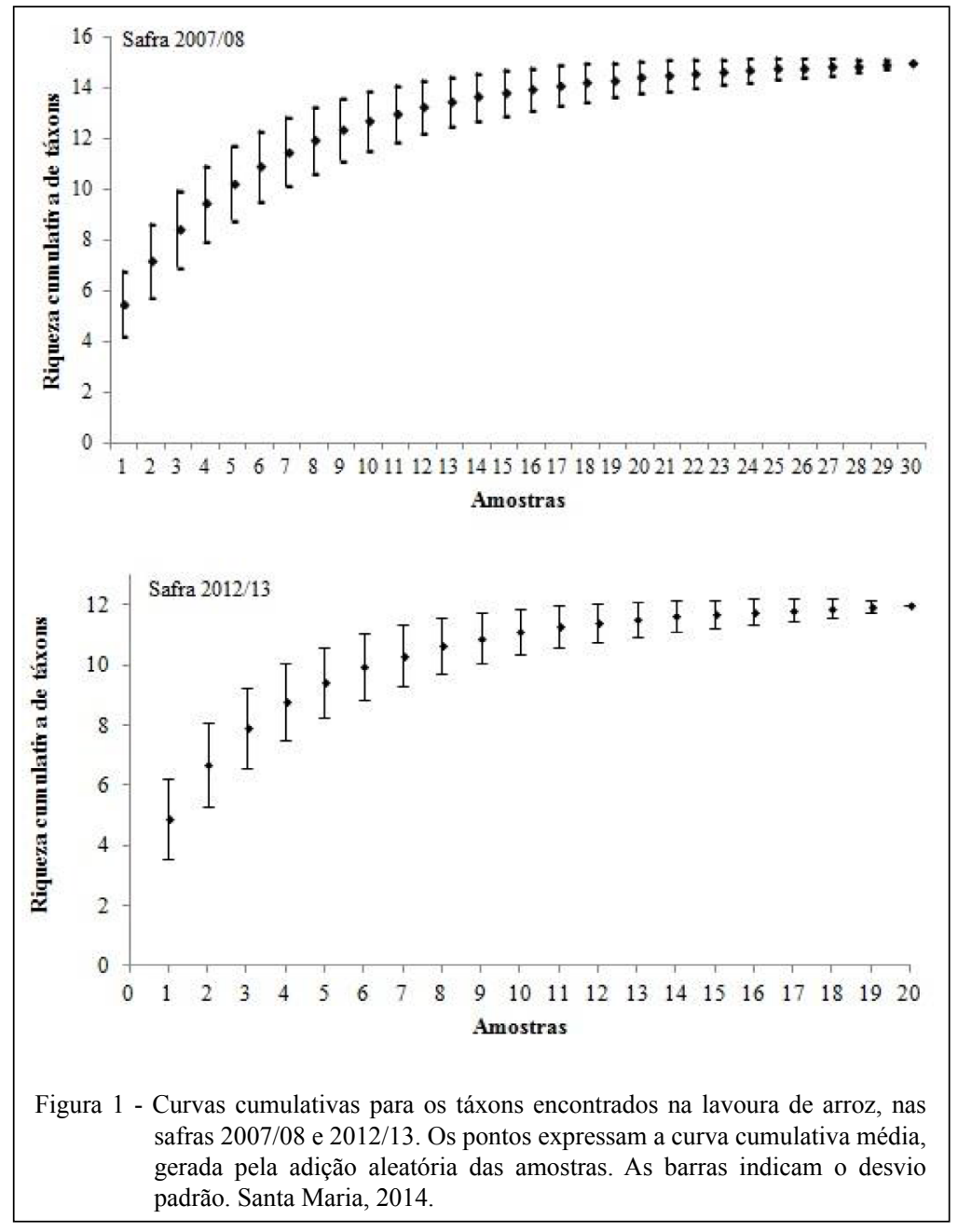

ferramentas importantes na adequação do esforço amostral (SILVA, 2012). Considerando o método de amostragem utilizado no presente trabalho, indica-se um número de 12 réplicas para se obter o maior número de famílias sem perda de informação, como suficiência amostral para estudos de impacto ambiental sobre a comunidade de macroinvertebrados bentônicos em áreas de cultivo de arroz irrigado.

\section{AGRADECIMENTOS}

Ao Conselho Nacional de Desenvolvimento Científico e Tecnológico (Edital Universal - MCTI/CNPq $n$. 14/2012) e à Coordenação de Aperfeiçoamento de Pessoal de Nível Superior (CAPES), pelo auxílio financeiro.

\section{REFERÊNCIAS}

BARBOUR, M.T. et al. Rapid bioassessment protocols for use in streams and wadeable rivers. Periphyton, benthic macroinvertebrates and fish. 2.ed. New York, 1999. Disponível em: <http://water.epa.gov/scitech/monitoring/rsl/bioassessment/>. Acesso em: 12 fev. 2013.

CAIN, S.A. The species-area curve. American Midland Naturalist. v.119, p.573-581, 1938. Disponível em: <http:// www.jstor.org/discover/10.2307/2420468?uid=3737664\&uid=2\&uid=4\&sid=21102586723843>. Acesso em: 20 jun. 2012.

COLWELL, R.K. EstimateS: statistical estimation of species richness and shared species from samples. Versão 8.2, 2009. Disponível em: $<$ http://viceroy.eeb.uconn.edu/estimates/>. Acesso em: 20 nov. 2012.

FERNÁNDEZ, H.R.; DOMÍNGUEZ, E. Guia para la determinación de los artrópodos bentónicos sudamericanos. Tucumán, Argentina: Imprenta Central de la UNT, 2001. 289p.

GOTELLI, N.J.; COLWELL. R.K. Estimating species richness. In: MAGURRAN, A.E.; McGILL, B.J. (Ed.). Biological diversity: frontiers in measurement and assessment. Oxford: Oxford University, 2010. p.39-54.

KUHLMANN, M.L. et al. Protocolo para o biomonitoramento com as comunidades bentônicas de rios e reservatórios do estado de São Paulo. São Paulo: CETESB, 2012. 113p. 
Disponível em: <http://www.cetesb.sp.gov.br/userfiles/file/agua/ aguas-superficiais/protocolo-biomonitoramento-2012.pdf $>$. Acesso em: 15 jul. 2013.

MELO, A.S. O que ganhamos 'confundindo' riqueza de espécies e equabilidade em um índice de diversidade? Biota Neotropica, v.8, n.3, p.21-27, 2008. Disponível em: <http://hdl.handle. net/10183/23298>. Acesso em: 17 out. 2014.

PILLAR, V.D. Suficiência amostral. In: BICUDO, C.E.M.; BICUDO, D.C. Amostragem em limnologia. São Carlos: RIMA, 2004. p. $25-43$

ROGER, P.A. et al. Biodiversity and sustainability of wetland rice production: role and potential of microorganisms and invertebrates. In: HAWKSWORTH, D.L. (Ed.). The biodiversity of microorganisms and invertebrates: its role in sustainable agriculture. UK: CAB International, 1991. p.117-136.

ROSENBERG, D.M.; RESH, V.H. Introduction to freshwater biomonitoring and benthic macroinvertebrates. In: ROSENBERG,
D.M.; RESH, V.H. (Eds). Freshwater biomonitoring and benthic macroinvertebrates. New York: Chapman \& Hall, 1993. 488p.

SCHILLING, A.C.; BATISTA, J.L.F. Curva de acumulação de espécies e suficiência amostral em florestas tropicais. Revista Brasileira de Botânica, v.31, n.1, p.179-187, 2008. Disponível em: $<$ http://dx.doi.org/10.1590/S0100-84042008000100016>. Acesso em: 15 out. 2014. doi: 10.1590/S0100-84042008000100016.

SILVA, D.R. de O. e. Estimativa de riqueza de macroinvertebrados bentônicos e a relação da composição de comunidades com componentes de meso-habitat em riachos de cabeceira no cerrado. 2012. 65f. Dissertação (Mestrado em Ecologia) - Programa de Pós-graduação em Ecologia, Conservação e Manejo de Vida Silvestre, Universidade Federal de Minas Gerais, MG.

SILVEIRA, L.F. et al. Para que servem os inventários de fauna? Estudos Avançados, v.24, n.68, p.173-207, 2010. Disponível em: $<$ http://dx.doi.org/10.1590/S0103-40142010000100015>. Acesso em: 20 out. 2014. doi: 10.1590/S0103-40142010000100015. 